\title{
Adiós a César Aguiar (1943-2011)
}

Recuerdo a César en la reunión de WAPOR de 1996, en Salt Lake City, con su característica sonrisa, buen humor y optimismo. Como buenos hermanos latinoamericanos que somos todos, desde el primer minuto nos llevamos como si tuviéramos años de tratarnos.

Con su energía y dinamismo empezamos a hacer planes de proyección y crecimiento. En aquel entonces, yo vivía en Ann Arbor, durante un año sabático en la universidad de Michigan, con Ron Inglehart. De inmediato, César aceptó formar parte del grupo de la Encuesta Mundial de Valores y levantar el estudio en Uruguay. Se había unido recientemente, junto con María Braun, a la red internacional que apenas iniciábamos de MORI.

Desde entonces, empezó a participar de manera activa en las reuniones que sosteníamos con regularidad, llevando siempre resultados positivos de su actividad en Uruguay y el Cono Sur. Otro rasgo que me impresionó de César fue su capacidad de identificar y rodearse de excelentes colaboradores, brillantes, trabajadores y valiosos, que eran una garantía de continuidad de su labor y que él cultivaba con esmero.

Tuve oportunidad de visitarlo brevemente en Montevideo hace doce años y comprobar la ebullición intelectual y empresarial que sus oficinas y sus colegas transmitían junto con él. En la tristeza de su partida a destiempo, me guardo el gusto de haberlo disfrutado por última vez en la reunión de WAPOR Latinoamérica en Querétaro hace casi tres años. 
Hay personajes que uno agradece a la vida haber tenido la oportunidad de conocer. De cruzar caminos y enriquecerse intelectualmente. Personajes que se distinguen por su entusiasmo, optimismo, empuje, alegría. César es sin duda, para todos los que lo conocimos y estimamos, uno de esos personajes.

Su contribución al desarrollo de las encuestas en Uruguay y el Cono Sur está hecha, y es una inspiración para sus colaboradores y amigos y para nuestra organización.

Miguel Basáñez Fletcher School, Tufts University Estados Unidos

El pasado 30 de diciembre murió en Montevideo César Aguiar, un referente fundamental para entender la construcción de las ciencias sociales aplicadas en la región durante el último cuarto de siglo.

A comienzos de la década de 1970, la dictadura uruguaya lo sorprendió joven, cuando había decidido dejar la abogacía por una, en aquellos tiempos, incierta y arriesgada apuesta por la sociología. Decidió vivir en su país, pero aprovechó su "insilio" para sentar las bases de dos instituciones que han sido referentes fundamentales de las ciencias sociales en Uruguay: CIEDUR (el Centro Interdisciplinario de Estudios para el Desarrollo) y Equipos Consultores, su amada criatura. Al mismo tiempo, construía su familia y ejercía la docencia en todos los lugares en que se lo permitían.

Ya durante aquellos años, fue transformándose en uno de los cientistas sociales más prestigiosos. Sus trabajos académicos sobre población, con un fuerte anclaje empírico, 
cuestionaron por primera vez en décadas los estereotipos corrientes sobre la población uruguaya. Sus estudios sobre estrategias de supervivencia mostraron la dinámica oculta de las familias uruguayas para enfrentar la crisis económica y social de los años 1970. Sus publicaciones sobre los clivajes políticos reposicionaron y redefinieron el concepto de "doble escena" que había sido desarrollado por Aldo Solari muchos años antes. Simultáneamente, desarrollaba su propia idea de ciencias sociales aplicadas y le daba forma en la construcción de Equipos Consultores.

La apertura democrática sería el momento en que su trabajo comenzaría a ser conocido de manera masiva, una popularidad que en buena medida devino de un pronóstico original, que fue capaz de realizar aun contradiciendo sus datos "crudos", la visión de la mayoría de sus colegas y sus propias preferencias. En aquel momento, todas las encuestas -incluyendo las que César dirigía- mostraban que la izquierda, representada por el Frente Amplio, lideraba con claridad la intención de voto en Montevideo. Todos le otorgaban la victoria. Pero estudiando los sesgos muestrales de sus propios estudios, y proyectando con algunas variables el comportamiento de los indecisos y las no respuestas, César concluyó que aunque la izquierda aparecía en primer lugar, lo más razonable era un triunfo del Partido Colorado. En tiempos en que los programas de computación no permitían ponderar las muestras ni imprimían cuadros mínimamente legibles para los legos, unos días antes de las elecciones presentó sus resultados en una reunión con clientes con el apoyo de un retroproyector y un conjunto de transparencias escritas a mano; la infidencia de un periodista allí presente hizo el resto. Vivió horas incómodas, porque sus colegas lo miraban con suspicacia y sus compañeros políticos lo acusaban de haberse vendido al enemigo, pero la realidad terminó 
mostrando que podía ser uno de sus observadores y analistas más lúcidos.

Esa creciente popularidad, asociada a sus dotes de profesor excepcional, le aseguró un rol fundamental en la creación de un nuevo perfil de las ciencias sociales en Uruguay. Esa visión estaba guiada por dos principios. Por un lado, la apertura a pensar que todos los temas, desde los que consideramos más profundos y trascendentes hasta los que a veces despreciamos por ser más cotidianos, eran una oportunidad para aprender de la realidad aportando un valor agregado desde las ciencias sociales y, a través de esa mirada, hacer mejor esa realidad y hacernos mejores nosotros mismos. Por otro lado, el convencimiento de que era posible desarrollar esa tarea bajo un formato empresarial, brindando servicios de calidad sin dejar en el camino ni los criterios técnicos ni la ética profesional.

Todos quienes estamos involucrados en estas áreas estaremos siempre agradecidos por el rol de César en el salto cualitativo que nuestras profesiones tuvieron en los últimos años. Fue uno de los grandes publicistas de nuestras disciplinas en Uruguay, no sólo por cómo ejerció, sino también porque nos mostró que una forma adecuada de comunicación de los conocimientos no tiene por qué abdicar del rigor ni la creatividad.

Esa visión pionera se expresó en el área de la opinión pública más allá de las fronteras de Uruguay. Fue un convencido de que era necesario desarrollar empresas locales y regionales independientes de los partidos y los gobiernos, pero con vínculos estrechos con la sociedad y la academia. Solía decir que la finalidad de los estudios de opinión pública era, ni más ni menos, mejorar la calidad de la política en nuestros países. Como militante convencido de la visión comparativa, buscó siempre intercambiar con otros colegas y generar bases de datos transnacionales. Es por eso que estuvo directamente involucrado en la creación 
de WAPOR en la región, así como fue su idea la generación de una encuesta comparativa que cristalizaría años después en el Latinobarómetro.

A lo largo del cuarto de siglo que compartimos laboralmente, algunas veces tuve formas diferentes de ver las cosas. Pero jamás dejé de aprovechar sus enseñanzas, y admirar su grandeza intelectual y su desprendimiento respecto a las ideas que generaba: en ese campo ha sido y será difícil encontrar personas tan generosas.

Toda su trayectoria profesional fue una manera de honrar la vida. Para quienes tuvimos la suerte de formarnos y trabajar con él, se fue uno de nuestros maestros. Vamos a extrañarlo mucho, y trataremos de recordarlo, todos los días, de una forma que estamos seguros que a César le hubiera gustado: con lo mejor de nuestra práctica profesional pensada y actuada de una manera abierta, creativa y sin complejos, ejercida con ética y complementada al menos con un poco de ese optimismo biológico que lo caracterizó siempre.

Agustín Canzani Director de la Fundación Líber Seregni Uruguay

Inteligente, talentoso, confiable, afable: son las palabras que me salen cuando pienso en César, nuestro querido colega y amigo. Mucho es lo que César contribuyó a las ciencias sociales y a la opinión pública en la región. Será siempre un referente ejemplar en nuestra profesión.

Marita Carballo Presidente de Kantar Latinoamérica Argentina 
Hablar de César Aguiar es una tarea complicada, nada sencilla, como era él. Compartí ocho años de trabajo con César, de él aprendí buena parte de las pocas cosas que sé, tanto en el área de la consultoría como de una visión global de la sociedad. Porque en César encontraba uno de esos pocos hombres con una gran capacidad de ver y pensar la sociedad globalmente, con una gran objetividad y sensibilidad social. Fue un hombre con una enorme capacidad de pensar hacia adelante, en una sociedad donde constantemente se mira hacia atrás, interpretando mal la historia, sin conocer el presente y sin pensar el futuro. Ello lo hacía una persona muy especial, no sólo como académico, sino también como analista, con una infinita capacidad de propuesta. En aquellos años que tuve el honor de trabajar con él, siempre nos sorprendía con alguna idea nueva, y cuando las cosas iban mal, César no se detenía a masticar el problema, simplemente proponía algo alternativo. Quienes trabajábamos con él solíamos decir "está tirando la pelota hacia adelante", y efectivamente era así, porque de hombres como él se construye el futuro de un país.

Analítico, soñador, hombre de acción. Construyó la principal consultora en ciencias sociales de Uruguay y, cuando ello le quedó chico, se trasladó afuera de fronteras. Posiblemente, el Uruguay es un país pequeño para contener hombres como César. Cuando se convirtió en el número uno, no se conformó, nunca se conformaba, nunca se rendía; cuando no había terminado con un proyecto, ya tenía dos o tres más pensados. Inquieto, dinámico, inteligente y emprendedor, nunca se dejó limitar por la mediocridad de un medio chato y estancado como el nuestro. Por el contrario, dijo e hizo cosas que algunos pensaron que eran locuras (sobre algunas de ellas también yo lo pensé). Siempre se ubicaba un paso adelante, asumía riesgos sin temor a equivocarse. 
Lógicamente, también podríamos mencionar algunos de los defectos de César, pero si no lo hago, no es porque haya fallecido, sino porque ninguno de ellos empequeñecía su figura. La desaparición de César me deja un gusto muy amargo, por él, por su familia y por mí, pero principalmente por este paisito que al perderlo no pierde un académico más, pierde ideas, pierde propuestas, pierde un ejemplo. Por lo menos, un ejemplo para mí.

Juan Carlos Doyenart

Interconsult

Uruguay

Cuesta aceptar que haya que despedir a César no estrechando su mano ni compartiendo un diálogo amigable, sino en un saludo postrero. Cuesta despedirlo con un adiós póstumo.

Esta no es por lo tanto una estricta despedida, sino más bien un homenaje. Un homenaje de un colega que sintió por él afecto y admiración, que valoró su producción profesional y su trabajo de emprendedor, que compartió con él y con otros colegas la ardua tarea de sentar las bases de la profesionalización de la investigación de opinión pública en el continente. Breve y austero, como él seguramente lo hubiera preferido. A mi generación profesional, sin César le está faltando algo irreemplazable.

Compartí con César cierta complicidad proveniente de que, más o menos al mismo tiempo, emprendimos proyectos parecidos. Crear consultoras, formar equipos y formar profesionales, comprometerse con los procesos políticos y sociales e intentar contribuir a ellos desde un lugar específico que antes no existía -la generación de información sistemática-, vivir con pasión el trabajo profesional y con 
la misma pasión el uso y abuso que la política hace de él, ese fue el costado de César que conocí. Nos enseñamos cosas mutuamente. Nos miramos a lo largo de los años a veces con un ojo y a veces con el otro, porque éramos colegas volcados con franqueza a compartir las experiencias, y también a veces competidores en un mercado duro, y siempre dispuestos a encontrarnos como amigos, disfrutar de un trago o una comida, divertirnos con las ocurrencias (las de él, siempre más ásperas e ingeniosas que las mías) y reflexionar sobre este mundo y lo que hacemos en él.

Quiero despedirme de César con la frase con la que cerró su ponencia en el encuentro sobre las encuestas y el cambio político, en Santiago, en la Universidad Diego Portales (recogida en el libro editado por Rodrigo Cordero La sociedad de la opinión): “De la calidad de la investigación y de los profesionales depende en buena medida la construcción de una opinión pública informada en una sociedad democrática".

Manuel Mora y Araujo Universidad Torcuato Di Tella Argentina

"Como un río de Leones su maravillosa fuerza

Como un torso de mármol su dibujada prudencia..." (Federico García Lorca).

César Aguiar contribuyó con su profesionalismo y excelencia al desarrollo de Latinobarómetro. Como colega, como miembro, y como encuestador.

Marta Lagos

Directora de MORI y Latinobarómetro

Chile 
Conocí a César en los late sixties en el atrio de la Universidad. Por allí transitaban, además de autoridades y funcionarios, los docentes y estudiantes de las facultades de Derecho y Ciencias Sociales, y los de Ciencias Económicas y Administración. Pertenecíamos entonces a facultades diferentes, pero compartíamos diversos espacios comunes, además del Paraninfo. Cuando uno entraba al edificio, por ejemplo, tenía a su derecha el Departamento de Publicaciones, dirigido por Eduardo Galeano, y a la izquierda, la vieja oficina de apuntes del Centro de Estudiantes de Derecho, convertida en una completa y buena librería que funcionaba bajo la égida de la Fundación de Cultura. Allí trabajaba yo, además de personajes como el inefable maoísta el "Gaucho" Moura, o el pintoresco anarco "Brasilero" Fuques -que luego dirigió la Fundación por años-, o Sergio Tomasso y Amabelia, socialistas de toda la vida, que venían de la vieja oficina de apuntes, y entre otros, Adolfo Wassen, que era tupamaro muy activo y terminó como rehén de la dictadura.

Por las escalinatas, bajaban -que es mucho más majestuoso que subir- el rector Cassinoni y el futuro autor de las principales letras del tango contemporáneo, colaborador de Astor Piazzolla, Horacio Ferrer, entre varios profesores ilustres del pasado y del porvenir.

Desde la planta baja, donde anidaba Ciencias Económicas, se asomaban futuros ministros y economistas de la talla de Enrique Iglesias, Federico Slinger, Ricardo Zerbino o Danilo Astori. Hasta el bedel de Facultad, el "Negro" Franklin Morales, se convirtió en el más "sesudo" de los periodistas deportivos. Esta era la clase de levadura en que se amasaban las cabecitas de esa época.

Gustavo Cosse, Vicente Cremanti y Jorge Lanzaro, con quienes estuvimos, entre muchos otros, compartiendo recuerdos y tristezas cuando despedimos a César, también 
estaban siempre por allí. Como Daniel Waksman, Mingo Carlevaro, Arturo Navarro Arana, y tantos otros.

En los años 1970, en dictadura y con la universidad intervenida por los militares, visitaba a César en la peculiar cooperativa de viviendas de la calle Pastoriza, que había levantado junto a otro grupo de católicos, con los que compartían hasta la misma cocina comunitaria.

En los meses del 74 en que yo fungía como responsable de una publicación del Partido Socialista en la clandestinidad, jamás me negó un comentario u opinión, algo a lo que no todos los que requerí se prestaron a hacer.

En los años 1980, ya convergimos en la Agencia de Publicidad (por entonces Pregón y más tarde Grey) de Francisco Vernazza. Ambos éramos, por distintas vías, muy buenos amigos de Pancho, y eso fue conduciendo nuestra relación a niveles superiores.

Para las elecciones del 84, que se hacían después de más de once años de férrea y despiadada dictadura militar, César tuvo la idea de hacer las primeras encuestas de opinión pública del país. Sólo existía en Uruguay una encuestadora que operaba bajo el paraguas del nombre Gallup, pero que en los hechos no reportaba a esa compañía internacional y se había desprestigiado mucho acompañando a los milicos cuando el plebiscito del 80.

César siempre pensaba y proyectaba en grande, y tenía siempre, también, una variedad de ideas nuevas "en cartera", que era una de sus características principales y más destacadas.

Así que, utilizando como plataforma de lanzamiento la Revista Búsqueda, sin cobrar y a puro pulmón, puso en el mercado una encuesta independiente, sólida técnicamente, sin apoyos de ningún sector político o empresarial, sin subsidios, pero con mucho coraje y visión; una encuesta que fue haciéndose conocida y creíble a fuerza de talento, trabajo, tenacidad y transparencia. La encuesta nacional 
de OP de Equipos Consultores. La madrina del saludable sistema de encuestas que posee hoy el Uruguay y que lo distingue de casi todos los demás países de nuestra América del Sur, sin mencionar lo que pasa, o más bien no pasa, en buena parte de todo el mundo.

En enero de 1985, con los resultados electorales a la vista y con el exitoso pronóstico publicado, César nos convocó a Pancho Vernazza y a mí a integrarnos a Equipos. Trabajamos juntos cerca de diez años. Siempre bajo la batuta de César, pero ya con aportes como los de Luis Eduardo González, Germán Rama, Agustín Canzani, Juan Carlos Doyenart y varios más.

César vivía empujando ideas y proyectos tan variados como trabajar en Buenos Aires, concretar la Fundación Colonia, hacer mediciones de audiencia, telemedios o la propia internacionalización de la empresa al convertirse en Equipos MORI.

Siempre innovando, siempre con generosidad, siempre incorporando gente joven al equipo, siempre emprendiendo, lo que no es necesariamente la característica más destacada de un académico. Cosa que César nunca dejó de ser. Lo era además a tiempo completo, vivía enseñando, pero sin escolasticismos, y siempre en contacto con la realidad. Poniendo a prueba sus ideas en concreto, jugándose el pellejo y sus mangos. Lo que no es tampoco muy habitual en la academia. Hay que reconocer que para eso lo ayudaba no sólo su talento, sus conocimientos y su imaginación, sino también su arte para vender ideas y proyectos. Estoy seguro de que él no se va a revolver en la tumba, sino que va a esbozar una sonrisa picarona cuando sepa que lo estoy definiendo, para variar, como el mejor vendedor que he conocido.

Sólo él podía transformar en tantos millones de dólares (a lo largo de casi treinta años de trabajo, no se apuren) sus ideas en el campo de las ciencias sociales aplicadas. 
Logrando que, en particular, en aquel Uruguay estancado y conservador, tanta gente estuviera dispuesta a jugarle unos buenos mangos a las patas de ese hombrecito bajo, gordito, peladito y sonriente. Eso sí, con el mejor aspecto de venerable. Y con una parla a la altura.

Para terminar, hace un par de años se le ocurrió volver a juntarse con Luis Eduardo González (hoy Cifra Consultores) para salir a exportar servicios de encuestas de opinión pública a otros mercados de América, aprovechando la oportunidad que daba el año electoral argentino y el prestigio acumulado por Equipos y Cifra.

Como siempre, se precisaba algún capital y algo más de experiencia empresaria; y trabajar con amigos y conocidos, por más diferencias que pudiéramos tener, siempre nos resultó atractivo, entonces pues volví a entrar. Así que tengo la alegría de despedir a este pequeño y venerable genio como socio fundador de su penúltima creación, la empresa Suma, dedicada a exportar talento uruguayo al resto de los países de América.

Ojalá que podamos estar a la altura del Venerable Maestro.

Carlos Lorenzo

Consorcio SUMA

Uruguay

Conocí a César en los años 1990. Nos reunieron nuestro común interés por la opinión pública y la investigación aplicada, así como nuestra asociación a WAPOR, la vinculación con MORI, la empresa que fundó el legendario Bob Worcester, y nuestra participación en el Latinobarómetro, donde ambos acompañamos desde el inicio a Marta Lagos. 
En las múltiples ocasiones que tuvimos oportunidad de charlar y trabajar juntos, me impresionó por su cultura y conocimiento de la realidad latinoamericana, así como por su capacidad para combinar una extraordinaria sensibilidad humana con una gran objetividad analítica. Recuerdo que la última vez que nos vimos, en una reunión del Latinobarómetro en Lima, conversamos mucho sobre el proceso electoral peruano y pronosticó correctamente el triunfo de Ollanta Humala. Pero no había que ser un investigador de la opinión pública para apreciar las cualidades personales de César. Su gentileza y su don de gentes eran visibles para todos. Se nos ha ido uno de los grandes de la investigación social y política de América Latina, pero sobre todo, un gran caballero.

Alfredo Torres IPSOS Apoyo

Perú

Tres veces vi a César peleando con patotas:

1. Con la patota idealista de 1984, unos veinte jovencitos envueltos en banderas del Frente Amplio caminado por 18 de Julio, a la salida del acto final, previo a las primeras elecciones posdictadura. Uno de ellos reconoció a César porque había sido su alumno, y nos paramos a conversar. El tema fue una imperdonable encuesta de Equipos, recién publicada, en la que pinchaba un globo sagrado: la victoria del Frente en Montevideo. Le dijeron traidor, le preguntaron quién le pagaba. César había sido asesor de Seregni, docente universitario destituido por los interventores de la dictadura y notorio firmante de varios manifiestos en tiempos ambiguos, en los que aún no se sabía qué era lo 
que la inteligencia militar iba a hacer con los nombres de los que se atrevían. Esos chiquilines probablemente nunca comprendieron lo injustos que habían sido. Cuando crecieron, pudieron organizar su reflexión política con más información que sus antecesores, porque gracias a tipos como César, la opinión pública aceptó las encuestas como un insumo importante y aprendió a distinguir el trabajo profesional de las operaciones políticas. Pero en el 84, tiempos calientes, no estaba aún claro que la verdad es más importante que la causa, aunque esta sea la de tus amores más profundos. César se había expuesto sabiendo lo que le esperaba, pero no le había temblado el pulso.

2. Con la patota adinerada de la televisión. Promediando los años 1990, Equipos empezó a medir las audiencias de televisión, y en poco tiempo, se transformó en la referencia indiscutida del mercado. Los avisadores y los canales negociaban los precios de cada programa con los informes de Equipos en la mano y, por tanto, se jugaba mucho dinero en los resultados. El asunto no les gustaba nada a los tres canales de Montevideo. Preferían el juego anterior, en el cual las audiencias eran un misterio, todo se vendía al mismo precio y se repartían el mercado amigablemente en cuasi tercios. Tenían un pool de compra de programación en el exterior y un pool de precios hacia el mercado interno, de modo que la oscuridad era un buen negocio para todos. Antes de empezar, César había sido advertido (por mí, entre otros) de que se metía en la jaula con los leones. Pero fue peor: excepto violencia física, los canales no ahorraron ninguna presión. Desde amenazar agencias de publicidad con el infierno si compraban las encuestas, hasta financiar mediciones truchas de otros proveedores, pasando por sueltos calumniosos en los diarios, en los que se insinuaba la venalidad de César, con nombre y apellido. Fueron años de presión continua con muchos costos para Equipos. Pero el muchachito de la película 
les ganó. No sé cómo, pero los hizo cansar. Años después, vino IBOPE y con tecnología e inversión barrió a Equipos del centro del mercado. Fue una derrota limpia que nunca amargó a César. Al fin de cuentas, fue él quien les aflojó la dentadura a los leones, que ya no pudieron morder a los que vinieron después.

3. Con la patota unipersonal de Sanguinetti. Tres años después de comenzar su primer mandato, todos los uruguayos teníamos que levantar la cabeza para mirar a nuestro “presidente sol”, Julio María Sanguinetti. No era fácil aguantarle la mirada: inteligente, culto, agudo en lo verbal, con una energía atlética, lucía como el capitán soñado de la restauración democrática. También tenía la mano pesada, por lo que no era recomendable causarle ningún disgusto. Por ejemplo, no era recomendable comenzar a hacer encuestas midiendo la popularidad y aprobación del gobierno. Equipos las inauguró allá por 1987, y como es natural, la secuencia temporal mostraba descensos en la cotización. El presidente enojado creyó que bastaba su autoridad intelectual para fulminar a los atrevidos, los acusó de brujería sociológica, porque “¿cómo es posible decir que la opinión de 400 encuestados representa a toda la opinión pública?". Unos meses después, cuando se publicó una nueva medición, ya alguien le había explicado a Sanguinetti algo sobre la teoría de las probabilidades como fundamento de las muestras, por lo que el desprecio del presidente viró a un argumento menos elegante: "No conozco a nadie que haya sido entrevistado, ¿de verdad harán las encuestas?". Y el asunto se cerró un trimestre después, cuando un periodista radial le preguntó a Sanguinetti si había leído la encuesta publicada ese día en el semanario Búsqueda, la publicación de política y economía más respetada del Uruguay. El presidente firmó la capitulación al disimulo, declarando: "No leo Búsqueda". Y de ahí en 
adelante, ningún actor político más o menos serio discutió en el Uruguay la legitimidad y el valor de las encuestas.

Por aquí, todo el mundo sabía que César volaba alto como intelectual y como emprendedor. Lo que a lo mejor no era tan público es que era un tipo valiente. Lo era de manera natural, casi distraída, sin tomarse en serio. Llevaba la valentía puesta como un rasgo más: era un petiso, gordito, sonriente y valiente.

Francisco Vernazza

Uruguay

I was deeply sorry to hear of Cesar's departure from this earth. He was a great man in a great country whose influence reached far. The work of Cesar in developing a culture of honesty in research throughout Latin America was impressive, as was his grasp of survey methodology. He developed and trained an outstanding cadre of enthusiastic colleagues and built a fine business. He was supportive of the wide use of the techniques of our trade and was assiduous in proselytising Uruguayan companies and companies operating in Uruguay / Paraguay / Columbia and certainly Argentina where he was so helpful to Maria. A good businessman, he did not put the pursuit of profit before integrity. I admired him greatly.

Professor Sir Robert Worcester Founder, MORI International 
Me resulta casi imposible resumir en unas pocas líneas todo lo que debería ser expresado sobre César Aguiar. Sus aportes al desarrollo del campo de la opinión pública en Uruguay, y en América Latina toda, apenas si muestran una de las tantas facetas en las que César desarrollaba sus talentos y virtudes. César estaba dotado de una profunda inteligencia, una capacidad innovadora como pocas, una gran audacia para llevar adelante nuevos proyectos (que a veces rozaba lo temerario), y una generosidad sin límites para el resto de los colegas en compartir sus ideas y su conocimiento, que se traslucía entre otras cosas en una actitud docente casi constante.

Todo esto combinado con dos elementos adicionales que marcaban su forma de ser y han marcado toda su trayectoria vital y profesional. Por un lado, una actitud lúdica verdaderamente original. Su frase de despedida de todos los días, e incluso en sus mails, era: “¡Divertirse!”. Y vaya si se divirtió en sus múltiples actividades dentro de las ciencias sociales, sin que esto signifique que haya claudicado en lo más mínimo de un rigor que era otra de sus características principales. Este fue un legado fundamental de César hacia las generaciones que formó: a su lado, aprendimos a querer, disfrutar y divertirnos con lo que hacemos, a ponerle pasión a la investigación, pero pasión combinada con rigor técnico. Y el segundo componente central que marcó su vida profesional, y marcó a todos los que compartimos con él estos años, fue una ética a prueba de balas. En estos casi veinte años que me tocó trabajar junto a César, fui testigo de algunas situaciones en las cuales hubo que tomar decisiones que involucraban dimensiones éticas, y en estos temas, César siempre fue absolutamente intransigente. Nunca le vi el menor titubeo. La ética con el cliente sí, pero, mucho más allá, la ética con la comunidad y la ética con los principios elementales de la actuación profesional (que en última instancia, son problemas "de almohada"). Estas 
cosas siempre estuvieron para César muy por encima de cualquier consideración de tipo económico.

Todos los que trabajamos junto a César estamos profundamente agradecidos de haber tenido ese privilegio. Su vida marcó de forma decisiva las nuestras. Su muerte deja un vacío en el campo de la opinión pública latinoamericana, pero aquellos que directa o indirectamente fuimos beneficiados con su sabiduría tenemos la responsabilidad de que su legado permanezca.

Ignacio Zuasnábar Director de Equipos MORI, Profesor de la Universidad Católica del Uruguay Uruguay 